\title{
Millimeter wave treatment inhibits the mitochondrion-dependent apoptosis pathway in chondrocytes
}

\author{
GUANGWEN WU ${ }^{1}$, THOMAS SFERRA ${ }^{3}$, XUZHENG CHEN $^{1}$, YOUQIN CHEN ${ }^{3}$, \\ MINGXIA WU ${ }^{1}$, HUIFENG XU ${ }^{2}$, JUN PENG $^{1}$ and XIANXIANG LIU ${ }^{1}$ \\ ${ }^{1}$ Academy of Integrative Medicine; ${ }^{2}$ Fujian Key Laboratory of Integrative Medicine on Geriatrics, \\ Fujian University of Traditional Chinese Medicine, Fuzhou 350108, P.R. China; \\ ${ }^{3}$ Department of Pediatrics, University of Oklahoma Health Sciences Center, Oklahoma City, OK 73190, USA
}

Received February 7, 2011; Accepted June 24, 2011

DOI: $10.3892 / \mathrm{mmr} .2011 .522$

\begin{abstract}
Millimeter wave (MW) is an electromagnetic wave with a wavelength between 1 and $10 \mathrm{~mm}$ and a frequency of 30-300 GHz that causes multiple biological effects, both locally and globally. MW has been widely used in clinical medicine. Although our previous work demonstrated that MW is capable of inhibiting sodium nitroprussiate (SNP)-induced apoptosis in chondrocytes, the precise mechanism of the antiapoptotic activity remains to be elucidated. The purpose of this study was to investigate the effects of MW in SNP-induced apoptotic chondrocytes. Sprague Dawley rat chondrocytes were isolated and cultured, and the cells were counted. Cell viability was evaluated using MTT assay. Cells were then treated with SNP and MW, and flow cytometry was used to detect apoptosis. Our results showed that MW treatment inhibited a SNP-induced mitochondrion-dependent pathway of apoptosis. MW treatment inhibited the loss of plasma membrane asymmetry (externalization of phosphatidylserine), collapse of mitochondrial membrane potential, and activation of caspase- 9 and caspase- 3 . Taken together, the results indicate that MW inhibits the mitochondrion-dependent pathway of apoptosis in chondrocytes and this may, in part, explain its clinical effect in the treatment of osteoarthritis.
\end{abstract}

\section{Introduction}

Osteoarthritis (OA) is characterized by a basic pathology of cartilage degeneration caused by the mutual effect of mechanical and biological factors. Cartilage is composed of chondrocytes that produce a large amount of extracellular matrix (ECM) including type II collagen fibers. Chondrocytes rapidly respond to changes in the joint microenvironment and

Correspondence to: Dr Jun Peng and Dr Xianxiang Liu, Academy of Integrative Medicine, Fujian University of Traditional Chinese Medicine, 1 Huatuo Road, Shangjie Minhou, Fuzhou 350108, P.R. China

E-mail: pjunlab@hotmail.com; liuxianxiang@163.com

Key words: chondrocyte, millimeter wave, apoptosis, mitochondria regulate the dynamic equilibrium between the degradation and synthesis of the ECM, which is crucial to the maintenance of cartilage function. Therefore, the functional changes of chondrocytes play significant roles by contributing to the degeneration of joint cartilage and thus the pathogenesis of OA.

Previous studies have shown that chondrocyte apoptosis is the vital mechanism of the degradation of cartilage $(1,2)$. Apoptosis is triggered either by intrinsic stimuli, such as cytokine deprivation and DNA damage, or by extrinsic stimuli, such as death ligand-receptor engagement. Both intrinsic and extrinsic signals eventually lead to the activation of cysteine-dependent aspartate-directed proteases (caspases) and nucleases, resulting in cell $(3,4)$. The best understood intrinsic apoptotic pathway is centered at the mitochondria. This apoptotic pathway, therefore, is referred to as mitochondrion-dependent apoptosis. It is the most common apoptotic pathway in vertebrate animal cells. A key commitment step in the induction of mitochondrion-dependent apoptosis is mitochondrial outer membrane permeabilization (MOMP), accompanied by the collapse of electrochemical gradient across the mitochondrial membrane. This event leads to the release of pro-apoptotic proteins, such as cytochrome $c$, which in turn trigger the activation of the caspase cascade (5-9).

A millimeter wave (MW) is an electromagnetic wave with a wavelength between 1 and $10 \mathrm{~mm}$ and a frequency of 30-300 GHz. Organisms produce coherent oscillations at $0.5 \times 10^{10}$ to $3 \times 10^{12}$ during metabolism similar to the frequency of MW. Therefore, the energy of MW can be absorbed by the organism through resonance. In turn, the energy may interfere with signal transduction and affect metabolism (10-13). MW has been shown to evoke multiple biological effects and is beneficial in the treatment of OA. Recently, we reported that MW inhibited sodium nitroprussiate (SNP)-induced apoptosis of chondrocytes (14). To further explore MW as a potential therapeutic agent in OA, the effect of MW on the mitochondrion-dependent apoptotic pathway in chondrocytes was investigated.

\section{Materials and methods}

Reagents. Fetal bovine serum (FBS), Dulbecco's modified Eagle's medium (DMEM), and trypsin were purchased from 
Hyclone Laboratories, Inc. (Logan, UT, USA). MTT, type-II collagenase and SNP were obtained from Sigma (St. Louis, MO, USA). An apoptosis assay (Annexin V-FITC Apoptosis Dection kit II) was provided by Becton-Dickinson (San Jose, CA, USA). A JC-1 mitochondrial membrane potential detection assay was obtained from Biotium, Inc. (Hayward, CA, USA). Caspase- 9 and caspase-3 colorimetric protease assays were obtained from Invitrogen Inc. (Grand Island, NY, USA). A flow cytometric Cytochrome $c$ release assay (InnoCyte kit) was obtained from EMD Chemicals, Inc. (Darmstadt, Germany).

Animals. Healthy and clean, 4-week-old, 90-110 g weight Sprague Dawley rats of either gender $(n=30)$ were purchased from Shanghai SLAC Laboratory Animal Inc., China [Laboratory Animal Use Certificate no. SCXK(SH)2007-0005] and raised in a sterile environment. Experiments involving the animals complied with Guidance Suggestions for the Care and Use of Laboratory Animals 2006 administered by the Ministry of Science and Technology, China (15).

Isolation and culture of rat chondrocytes. Rat chondrocytes were isolated and cultured as previously described (14). The cells used in these experiments were counted with a hemocytometer and adjusted to $10^{4}-10^{6} \mathrm{cell} / \mathrm{s} / \mathrm{ml}$.

Evaluation of cell viability by MTT assay. Cell viability was assessed by the 3-(4,5-dimethylthiazol-2-yl)-2, 5-diphenyltetrazolium bromide (MTT) colorimetric assay. P2 chondrocytes were seeded into 96-well plates at a density of $1.0 \times 10^{4}$ cells/well in $0.1 \mathrm{ml}$ of medium. The cells were treated with various concentrations of SNP for 24 or $48 \mathrm{~h}$. At the end of the treatment, $100 \mu 1$ MTT [0.5 mg/ml in phosphate-buffered saline (PBS)] were added to each well and the samples were incubated for an additional $4 \mathrm{~h}$ at $37^{\circ} \mathrm{C}$. The purple-blue MTT formazan precipitate was dissolved in $100 \mu \mathrm{l}$ DMSO. The absorbance was measured at $490 \mathrm{~nm}$ using an Elx $808^{\mathrm{TM}}$ absorbance microplate reader (BioTek Instruments, Inc., VT, USA). The relative cell viability was expressed as the ratio (\%) of the absorbance in the experimental wells to that of the control wells (normal culture without treatment). Following this, the IC50 value (cytotoxic concentration for 50\% cell death) was determined from the dose-response curve.

MW stimulation. A KFA-100A MW therapeutic instrument (incidence power density: $4 \mathrm{mw} / \mathrm{cm}^{2}$; wavelength coverage: $7.5-10.0 \mathrm{~mm}$ ) was manufactured by Beijing Zhongcheng KangFu Technology Co., Ltd. (Beijing, China). As shown in Fig. 1, the detecting head of the instrument (radiation source) was positioned $30 \mathrm{~mm}$ above the cells (cultured in MW treatment group), which ensured that the cells were within the radiation area of $33.2 \pm 3 \times 45.6 \pm 4 \mathrm{~mm}$.

Cell treatment. $\mathrm{P} 2$ chondrocytes were digested with $0.25 \%$ trypsin and incubated in $25 \mathrm{ml}$ culture flasks at a density of $1 \times 10^{5}$ cells $/ \mathrm{ml}$ in $5 \mathrm{ml}$ of medium and in 6-well plates at a density of $1 \times 10^{5}$ cells $/ \mathrm{ml}$ in $2 \mathrm{ml}$ of medium for $24 \mathrm{~h}$. Cells were subsequently divided into 4 groups: control group (normal culture without treatment); $1 \mathrm{mM} \mathrm{SNP}$ group (treatment with $1 \mathrm{mM}$ SNP and no MW signal); $1 \mathrm{mM} \mathrm{SNP} \mathrm{+}$ MW 30 group (treatment with $1 \mathrm{mM} \mathrm{SNP}$ and MW signal
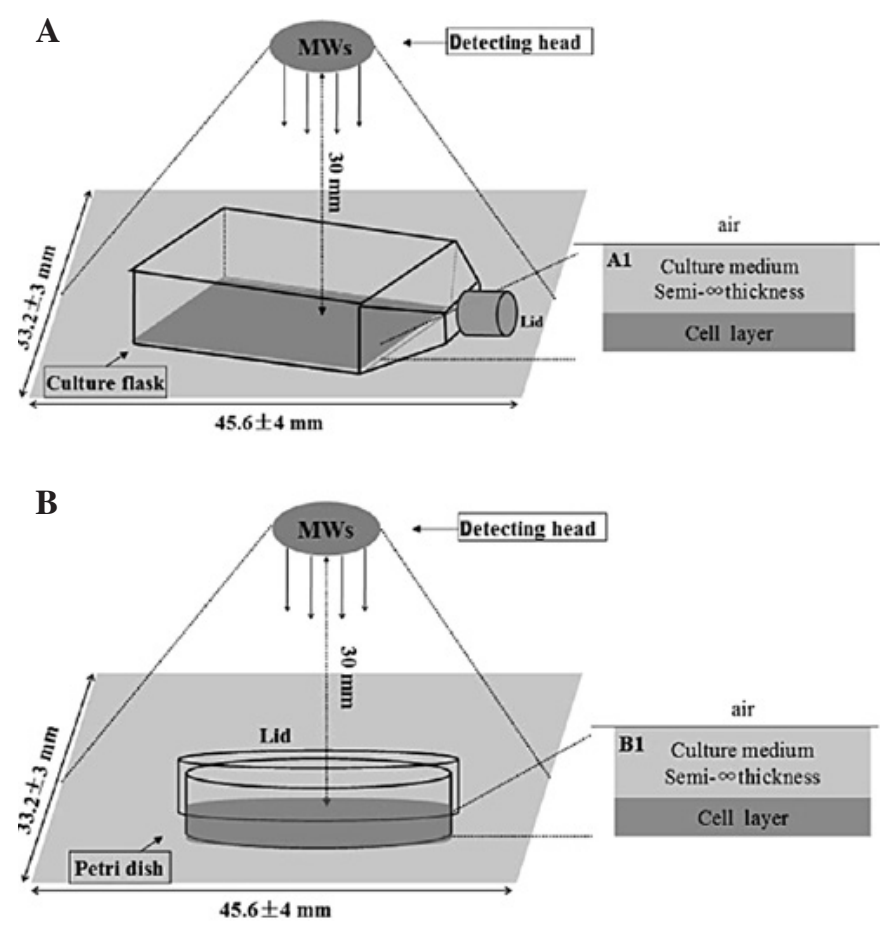

Figure 1. MW treatment method. (A and B) Schematic representation of the MWs far field exposure set-up used for investigating MW-induced in vitro effects on cell systems cultured in $25-\mathrm{ml}$ culture flasks and $3.5-\mathrm{cm}$ petri dishes. Enlargement of the culture flasks and petri dish showing all the dielectric interfaces through which millimeter radiation propagates. (A1 and B1) The multiple stratified dielectric system was used to model the reflection properties of the Culture flask and Petri dish cell culture system.

for $30 \mathrm{~min}$ on 3 consecutive days); and $1 \mathrm{mM}$ SNP + MW 60 group (treatment with $1 \mathrm{mM}$ SNP and MW signal for 60 min on 3 consecutive days).

Detection of apoptosis by flow cytometry with Annexin V/PI staining. Following treatment with SNP and MW, apoptosis of chondrocytes was determined by flow cytometry (FCM) using a fluorescence-activated cell sorting (FACS) caliber (FACSCalibur, Becton-Dickinson) and the Annexin V-FITC apoptosis detection kit II. Staining was performed according to the manufacturer's instructions and as we previously described (16). The percentage of cells in early apoptosis was calculated by Annexin V-positivity and propidium idodide (PI)-negativity, while the percentage of cells in late apoptosis was calculated by Annexin V-positivity and PI-positivity.

Measurement of mitochondrial membrane potential $(\Delta \Psi)$ using flow cytometry with JC-1 staining. To evaluate for the loss of mitochondrial membrane potential, a hallmark of apoptosis, cells were stained with the fluorescent dye JC-1. In this experiment, $1 \times 10^{6}$ treated chondrocytes were resuspended after trypsinization in $0.5 \mathrm{ml}$ of medium and incubated with $10 \mu \mathrm{g} / \mathrm{ml}$ of $\mathrm{JC}-1$ at $37^{\circ} \mathrm{C}, 5 \% \mathrm{CO}_{2}$, for $15 \mathrm{~min}$. Red and green fluorescence emissions were analyzed by flow cytometry.

Analysis of cytochrome c staining by flow cytometry. After treatment with SNP and MW for $24 \mathrm{~h}$, cytochrome $c$ release was determined by flow cytometry using the InnoCyte ${ }^{\mathrm{TM}}$ flow cytometric cytochrome $c$ release kit. Cytochrome $c$ staining 




Figure 2. Effect of SNP on chondrocyte viability. Chondrocytes were treated with the indicated concentrations of SNP for 24 or $48 \mathrm{~h}$. Cell viability was determined by the MTT assay. Data are shown as the mean \pm SEM (standard error mean) of at least three independent experiments. ${ }^{*} \mathrm{P}<0.05,{ }^{* *} \mathrm{P}<0.01$, compared with the untreated control.

was performed according to the manufacturer's instructions. The results were analyzed using the mean fluorescent intensity (MFI).

Analysis of caspase activation. The activity of caspase-9 and caspase-3 were determined with a colorimetric assay using a colorimetric protease assay, following the manufacturer's instructions and our previous description (16). Briefly, after treatment with SNP or MW for $24 \mathrm{~h}$, chondrocytes were lysed with lysis buffer for $10 \mathrm{~min}$ on ice according to the manufacturer's instructions. The lysed cells were centrifuged at $10,000 \mathrm{x} \mathrm{g}$ for $10 \mathrm{~min}$. An aliquot $(100 \mu \mathrm{g})$ of the protein was incubated with $50 \mu \mathrm{l}$ of the colorimetric tetrapeptides, LeuGlu-His-Asp (LEHD)-pNA (specific substrate of caspase-9) or Asp-Glue-Val-Asp (DEVD)-pNA (specific substrate of caspase-3) at $37^{\circ} \mathrm{C}$ in the dark for $2 \mathrm{~h}$. Samples were read at $405 \mathrm{~nm}$ in an absorbance microplate reader (Elx808, BioTek Instruments, Inc.). The data were normalized to the activity of the caspases in control cells and represented as 'fold of control'.

Statistical analysis. Data are the means of three determinations. Data were analyzed using the SPSS package for Windows (version 13.0). Statistical analysis of the data was performed with the Student's t-test and ANOVA. Differences with $\mathrm{P}<0.05$ were considered to be statistically significant.

\section{Results}

Effect of MW on the SNP-induced apoptosis of chondrocytes. We used SNP, a nitric oxide (NO) donor, to induce NOmediated apoptosis in chondrocytes (17). To optimize our experimental conditions, we evaluated the effect of different amounts of SNP on chondrocyte survival. As shown in Fig. 2, SNP decreased the viability of chondrocytes in a time- and dose-dependent manner. The concentration for $50 \%$ inhibition (IC50) of cell viability was $1.145 \pm 0.073$ and $0.566 \pm 0.061 \mathrm{mM}$ for 24 and $48 \mathrm{~h}$, respectively. Therefore, $1 \mathrm{mM} \mathrm{SNP}$ and $24 \mathrm{~h}$ were used as the inducing concentration and time factors in the subsequent experiments. Chondrocyte apoptosis was determined using Annexin-V/PI staining followed by FCM analysis. In this assay, the Annexin V/PI double-negative population (labeled as LL quadrant, Fig. 3) indicates viable cells; Annexin V-positive/PI-negative or Annexin V/PI double-positive population (labeled as LR or UR, Fig. 3) are cells undergoing early or late apoptosis, respectively. As shown in Fig. 3A and B, the percentage of cells undergoing early apoptosis was $1.58 \pm 0.14,30.54 \pm 0.78,23.25 \pm 0.49$, and $17.45 \pm 0.75 \%$ in the control, $1 \mathrm{mM} \mathrm{SNP,} 1 \mathrm{mM} \mathrm{SNP}+\mathrm{MW}$ 30 and $1 \mathrm{mM} \mathrm{SNP}+\mathrm{MW} 60$ groups, respectively. The $1 \mathrm{mM}$ SNP group showed significantly more apoptosis than the control group $(\mathrm{P}<0.01)$. Both the $1 \mathrm{mM} \mathrm{SNP}+\mathrm{MW} 30$ and $1 \mathrm{mM}$ SNP + MW 60 groups showed significantly less apoptosis compared with the $1 \mathrm{mM}$ SNP group $(\mathrm{P}<0.05)$. A dose-dependent effect was evident as the group that received the longest exposure to the MW signal (1 mM SNP + MW 60 group) demonstrated the least number of apoptotic cells $(\mathrm{P}<0.05$ vs. $1 \mathrm{mM} \mathrm{SNP}+\mathrm{MW} 30)$.

Effect of $M W$ on the loss of mitochondrial potential $(\Delta \psi)$. FCM analysis with JC-1 staining was then used to examine the change in mitochondrial membrane potential $\Delta \psi$ following MW treatment. JC-1 accumulates in normal intact mitochondria and forms aggregates. Aggregation of JC-1 results in red fluorescence $(590 \mathrm{~nm}, \mathrm{FL}-2)$. The intensity of the fluorescence is proportional to the mitochondrial membrane potential. Loss of membrane potential leads to the conversion of JC-1 to its monomeric form within the cytoplasm. This results in green fluorescence (529 nm, FL-1). Representative results from a single JC-1 assay for each treament group are shown in Fig. 4A. As shown in Fig. 4B, the percentage of apoptotic cells (LR quadrant) was 6.01 $\pm 1.78,53.83 \pm 3.13$, $29.60 \pm 2.12$ and $15.01 \pm 1.89 \%$ in the control group, $1 \mathrm{mM}$ SNP, $1 \mathrm{mM} \mathrm{SNP} \mathrm{+} \mathrm{MW} 30$ and $1 \mathrm{mM} \mathrm{SNP} \mathrm{+} \mathrm{MW} 60$ groups, respectively. Compared with the control group, the $1 \mathrm{mM} \mathrm{SNP}$ group had a greater number of cells with low red fluorescence [FL-2 (LR)] $(\mathrm{P}<0.01)$, indicative of a reduction in the $\Delta \psi$ and the induction of apoptosis. Both the $1 \mathrm{mM}$ SNP + MW 30 and $1 \mathrm{mM}$ SNP + MW 60 groups had significantly fewer cells with low red fluorescence [FL-2 $(\mathrm{LR})](\mathrm{P}<0.05)$ as compared to the $1 \mathrm{mM}$ SNP group. This indicates that MW treatment inhibited SNP-induced apoptosis. A dose-dependent effect was evident as the $1 \mathrm{mM} \mathrm{SNP}+\mathrm{MW} 60$ group demonstrated the least number of cells with low red fluorescence $(\mathrm{P}<0.05$ vs. $1 \mathrm{mM}$ SNP + MW 30).

Effect of $M W$ on the release of cytochrome $c$. To determine the effect of MW treatment on the release of cytochrome $c$ from the mitochondria, a FCM assay was used based on the selective permeabilization of the cell membrane while leaving the mitochondrial membrane intact. In this assay, viable cells exhibit staining of cytochrome $c$, whereas cells committed to the apoptotic process do not stain since they release cytochrome $c$ from the mitochondria to the cytosol and, subsequently, out of the permeabilized cell. As shown in Fig. 5, the MFI was $45.7 \pm 1.178,24.837 \pm 1.856$, 30.327 \pm 1.273 , and $39.157 \pm 1.067$ in the control, $1 \mathrm{mM}$ SNP, $1 \mathrm{mM}$ SNP + MW 30 and 1 mM SNP + MW 60 groups, respectively. Compared with the control group, $1 \mathrm{mM}$ SNP group had significantly reduced cytochrome $c(\mathrm{P}<0.01)$ staining. Both the $1 \mathrm{mM} \mathrm{SNP}+\mathrm{MW} 30$ and $1 \mathrm{mM} \mathrm{SNP}+\mathrm{MW} 60$ groups release significantly less cytochrome $c$ compared with the $1 \mathrm{mM}$ SNP group $(\mathrm{P}<0.05)$. A dose-dependent effect was 
A



Annexin V

B

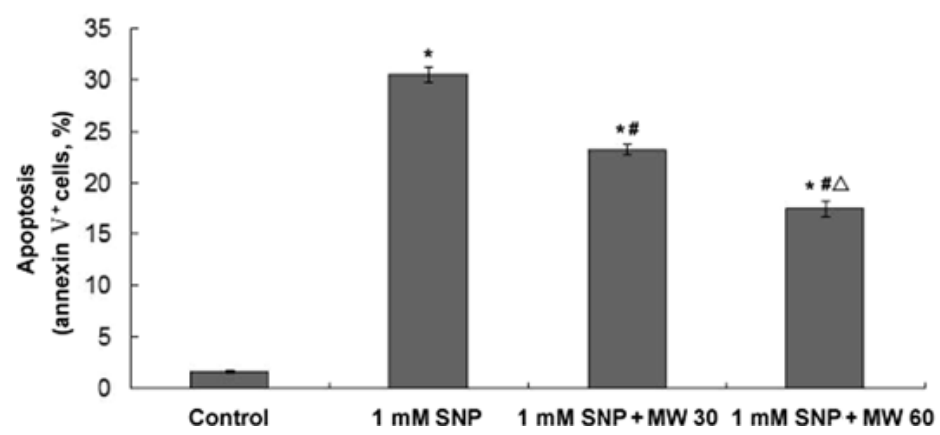

Figure 3. Effect of MW on the apoptosis of chondrocytes. After treatment with or without $1 \mathrm{mM} \mathrm{SNP}$ or MW for $24 \mathrm{~h}$ chondrocytes were collected and stained with Annexin V/PI followed by FCM analysis. (A) Representative FCM analysis scatter-grams of Annexin V/PI staining show four different cell populations labeled as: double-negative stained cells (LL, lower left) representing the live cell population; Annexin V-positive/PI-negative stained cells (LR, lower right) and Annexin V/PI double-positive stained cells (UR, upper right) representing early apoptosis and late apoptosis, respectively; Annexin V-negative and PI-positive stained cells (UL, upper left) representing dead cells. (B) FCM results are expressed as the mean \pm SD of three independent experiments. "P<0.01, compared with the control group. ${ }^{\#} \mathrm{P}<0.05$, compared with the $1 \mathrm{mM}$ SNP group. ${ }^{\wedge} \mathrm{P}<0.05$, compared with the $1 \mathrm{mM} \mathrm{SNP}+\mathrm{MW} 30$ group.

A

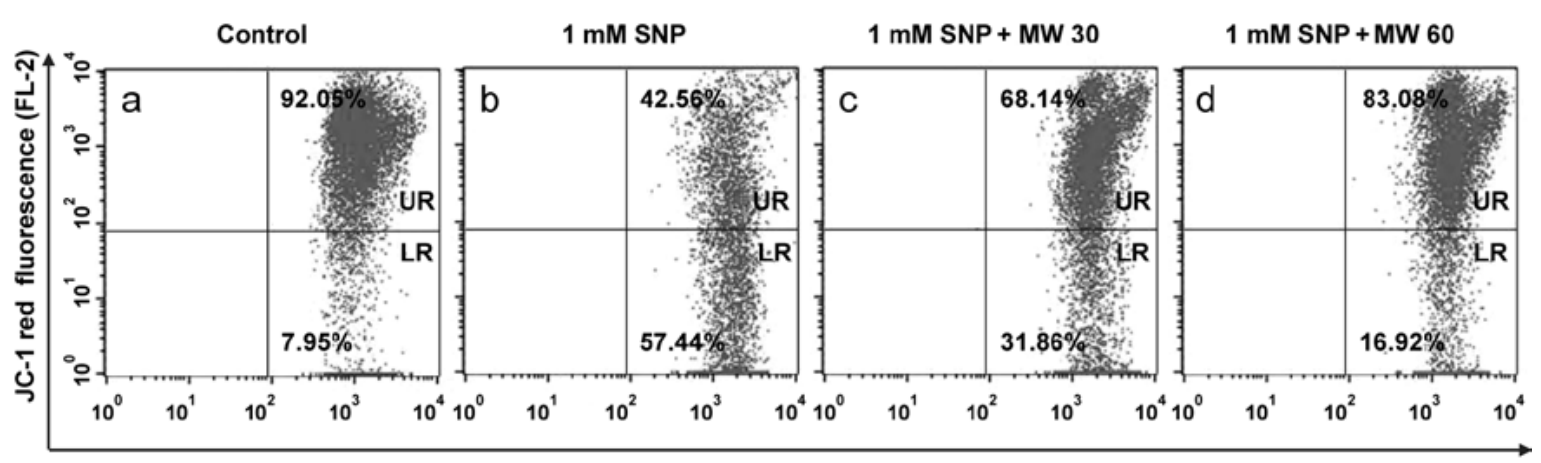

JC-1 green fluorescence (FL-1)

B



Figure 4. Effect of MW on the loss of mitochondrial membrane potential in chondrocytes. Chondrocytes were treated with or without $1 \mathrm{mM}$ SNP or MW for $24 \mathrm{~h}$ and stained with JC-1. (A) Representative results from the JC-1 FCM analysis. (a) JC-1 fluorescence was noted in both the FL-2 and FL-1 channels (UR) in the majority of untreated control cells. A small percentage of the control cells fluoresced in the FL-1 channel and lacked fluorescence in the FL-2 channel (LR). (b) There was a greater number of cells with low red (FL-2) fluorescence (LR), indicative of an alteration in the $\Delta \psi$, in the SNP-induced group due to undergo apoptosis. (c and d) MW treatment resulted in a reduction in the number cells with low red fluorescence (LR) as compared to SNP-treated cells, indicative of inhibition of the alteration in $\Delta \psi$. (B) Results are expressed as the mean \pm SD of three independent experiments. "P<0.01, compared with the control group. ${ }^{~} \mathrm{P}<0.05$, compared with the $1 \mathrm{mM}$ SNP group. ${ }^{\triangle} \mathrm{P}<0.05$, compared with the $1 \mathrm{mM} \mathrm{SNP}+\mathrm{MW} 30$ group. 


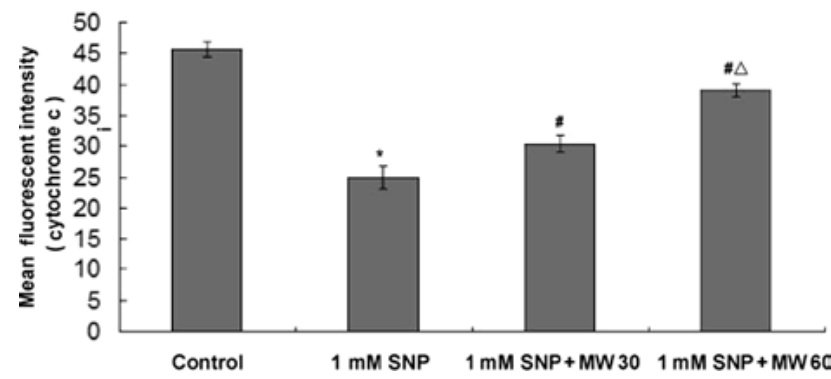

Figure 5. Effect of MW on the release of cytochrome $c$ in chondrocytes. Cells were treated with or without $1 \mathrm{mM} \mathrm{SNP}$ or MW for $24 \mathrm{~h}$. The MFI of cytochrome $c$ positively stained cells was determined using FCM analysis Data are the mean \pm SD from at least three independent experiments ${ }^{*} \mathrm{P}<0.01$, compared with the control group. ${ }^{\#} \mathrm{P}<0.05$, compared with the $1 \mathrm{mM}$ SNP group. ${ }^{\triangle} \mathrm{P}<0.05$, compared with the $1 \mathrm{mM}$ SNP + MW 30 group.



Figure 6. Effect of MW on the activity of caspase-9 in chondrocytes. Cells were treated with or without $1 \mathrm{mM} \mathrm{SNP}$ or MW for $24 \mathrm{~h}$. Caspase- 9 activity was determined by a colorimetric assay. The data was normalized to the caspase activities within control untreated cells and represented as 'fold of control'. Data are the mean \pm SD from at least three independent experiments. ${ }^{*} \mathrm{P}<0.01$, compared with the control group. ${ }^{\#} \mathrm{P}<0.05$, compared with the $1 \mathrm{mM}$ SNP group. ${ }^{\triangle} \mathrm{P}<0.05$, compared with the $1 \mathrm{mM} \mathrm{SNP}+\mathrm{MW} 30$ group.



Figure 7. Effect of MW on the activity of Caspase-3 in chondrocytes. Cells were treated with or without $1 \mathrm{mM} \mathrm{SNP}$ or MW for $24 \mathrm{~h}$. Caspase-3 activity was determined by a colorimetric assay. The data were normalized to the caspase activities within the control untreated cells and are represented as 'fold of control'. Data are the mean $\pm \mathrm{SD}$ of at least three independent experiments. ${ }^{*} \mathrm{P}<0.01$, significant vs. control group. ${ }^{\text {" }} \mathrm{P}<0.05$, compared with the $1 \mathrm{mM}$ SNP group. ${ }^{\triangle} \mathrm{P}<0.05$, compared with the $1 \mathrm{mM} \mathrm{SNP}+\mathrm{MW} 30$ group.

evident (1 mM SNP + MW 60 group vs. $1 \mathrm{mM} \mathrm{SNP} \mathrm{+} \mathrm{MW}$ 30 group, $\mathrm{P}<0.05)$.

Effect of MW on the activation of caspase-9 and caspase-3. To identify the downstream effectors in the apoptotic signaling pathway, the activation of caspases- 9 and caspases- 3 were examined by a colorimetric assay using specific chromophores, LEHD-pNA (specific substrate of caspase-9) and DEVD-pNA (specific substrate of caspase-3). As shown in Figs. 6-7, MW treatment significantly and dose-dependently inhibited activation of caspase- 9 and caspase- 3 in P2 chondrocytes $(\mathrm{P}<0.01$, vs. untreated control cells $)$.

\section{Discussion}

Osteoarthritis (OA), the most common age-related cartilage and joint disorder (18), is a slowly progressive degenerative disease characterized by degradation of the extracellular matrix and cell death resulting in a gradual loss of articular cartilage integrity $(19,20)$. The only cell type present in mature cartilage is the chondrocyte. This cell type is responsible for repairing damaged cartilage tissue. We previously demonstrated that chondrocytes are obtained by mechanical and chemical isolation methods and that the purity of the isolated chondrocytes is high $(14,21)$. This process provides allowed for the investigation of the effect of MW on chondrocyte apoptosis.

NO is a crucial physiological and pathological signaling molecule. It can be produced by inflammatory stimuli, nitroso compounds, and exogenous donors such as SNP. NO inhibits the proliferation of chondrocytes and induces chondrocyte apoptosis. Thus, NO induces cartilage damage and impairs cartilage repair (17). To develop a model in which to study the effect of MW treatment on chondrocyte apoptosis, we investigated the dose- and time- effect relationship of SNP on the induction of chondrocyte apoptosis. We observed that SNP decreased the viability of P2 chondrocytes in a dose- and time-dependent manner. Based on these results, we selected the dose of $1 \mathrm{mM} \mathrm{SNP}$ and the time point of $24 \mathrm{~h}$ to complete the described studies.

The loss of plasma membrane asymmetry is one of the morphologic characteristics of the apoptotic program. In apoptotic cells, the membrane phospholipid phosphatidylserine (PS) is translocated from the inner to the outer leaflet of the plasma membrane, thereby exposing PS to the external cell environment. Annexin $\mathrm{V}$ is a $35-36 \mathrm{kDa} \mathrm{Ca}^{2+}$-dependent phospholipid-binding protein that has a high affinity for PS. Annexin $\mathrm{V}$ binds to cells with exposed PS. Therefore, we used flow cytometry with annexin-V staining to examine the effect of MW on the apoptosis of chondrocytes. We observed that $1 \mathrm{mM} \mathrm{SNP}$ induced apoptosis after $24 \mathrm{~h}$ of exposure (Fig. 2), confirming the feasibility of using SNP-induced apoptosis of chondrocytes to study the effect of MW treatment. Using this cell culture model and assay, MW was found to inhibit SNP-induced apoptosis (Fig. 3A and B). Moreover, a dose-dependent effect was evident as longer exposure to the MW signal had a greater effect in the inhibition of apoptosis $(\mathrm{P}<0.05)$.

The loss of mitochondrial membrane potential $(\Delta \psi)$ is a hallmark of apoptosis. It is an early event preceding phosphatidylserine externalization and coincides with caspase activation $(22,23)$. We used a JC-1 dye-based assay to evaluate mitochondrial membrane potential. In healthy cells, the dye stains the mitochondria fluorescent red (24). The negative charge established by the intact mitochondrial membrane potential allows this lipophilic dye, bearing a delocalized positive charge, to enter the mitochondrial matrix where it accumulates. When the critical concentration is exceeded, 
J-aggregates form. These aggregates are fluorescent red $(590 \mathrm{~nm})$. In apoptotic cells, the mitochondrial membrane potential collapses, and $\mathrm{JC}-1$ cannot accumulate within the mitochondria. In these cells, JC-1 remains in the cytoplasm in a green fluorescent monomeric form. JC-1-stained apoptotic cells, having primarily green fluorescence $(530 \mathrm{~nm})$, are easily differentiated from healthy cells that have red and green fluorescence (25). Using FCM, healthy cells with red JC-1 aggregates are detected in the FL-2 channel and apoptotic cells with green JC-1 monomers are detected in the FL-1 channel. Thus, JC-1-stained cells that fluoresce in the FL-2 and FL-1 channels (UR quadrant) carry mitochondria with a polarized $\Delta \psi$, whreas JC-1-stained cells that fluoresce in the FL-1 channel and not in the FL-2 channel (LR quadrant) carry mitochondria with a depolarized $\Delta \psi$. In the present study, SNP was found to induce mitochondrial membrane depolarization indicative of apoptosis in chondrocytes and MW treatment inhibits this effect in a dose-dependent manner.

The mitochondrion-dependent pathway is the most common apoptotic pathway in vertebrate animal cells. Mitochondrial outer membrane permeabilization (MOMP) accompanied by the collapse of electrochemical gradient across the mitochondrial membrane is a key commitment step in the induction of mitochondrion-dependent apoptosis. This is the point of convergence for a large variety of intracellular apoptotic signaling pathways that eventually lead to the release of pro-apoptotic proteins from the mitochondrial intermembrane space, including cytochrome $c$, Smac/DIABLO, and Omi/ HtrA2. Released cytochrome $c$ activates APAF-1, which oligomerizes to form an apoptosome. This structure, in turn, recruits and activates caspase-9. Activated caspase-9 cleaves and activates executioner caspases, such as caspase-3, and eventually results in apoptosis $(22,23,26)$. Therefore, to evaluate the effect on MW on the mitochondrion-dependent apoptosis pathway, we evaluated the release of cytochrome $c$ and the activation of caspase- 9 and caspase- 3 . MW treatment dose-dependently inhibited the release of cytochrome $c$ and the activation of caspase- 9 and caspase- 3 .

In conclusion, our data show that MW treatment is capable of inhibiting SNP-induced mitochondrion-dependent apoptosis in chondrocytes. Furthermore, we noted that this effect was dose-dependent. However, our findings are limited to the inhibitory effect of MW treatment on SNP-induced apoptosis. Thus, whether an MW can inhibit OA or the natural apoptosis of chondrocytes remains to be evaluated.

\section{Acknowledgements}

This work was supported by the Developmental Fund of Chen Keji Integrative Medicine (CKJ2010023) and 2008 Fujian Province Small Creations and Projects (no.1).

\section{References}

1. Bar-Yehuda S, Rath-Wolfson L, Del Valle L, et al: Induction of an antiinflammatory effect and prevention of cartilage damage in rat knee osteoarthritis by CF101 treatment. Arthritis Rheum 10: 3061-3071, 2009.

2. Legendre F, Heuze A, Boukerrouche K, et al: Rhein, the metabolite of diacerhein, reduces the proliferation of osteoarthritic chondrocytes and synoviocytes without inducing apoptosis. Scand J Rheumatol 2: 104-111, 2009.
3. Cory S and Adams JM: The Bcl-2 family: regulators of the cellular life-of-death switch. Nat Rev Cancer 2: 647-656, 2002.

4. Borner $\mathrm{C}$ : Bcl-2 family members: integrators of survival and death. Biochim Biophys Acta 1644: 71-72, 2004.

5. Wei MC, Lindsten T, Mootha VK, et al: tBid, a membranetargeted death ligand, oligomerizes Bak to release Cytochrome $c$. Genes Dev 14: 2060-2071, 2000.

6. Antonsson B, Montessuit S, Lauper S, Eskes R and Martinou JC: Bax oligomerization is required for channel-forming activity in liposomes and to trigger Cytochrome $c$ release from mitochondria. Biochem J 345: 271-278, 2000.

7. Jürgensmeier JM, Xie Z, Deveraux Q, Ellerby L, Bredesen D and Reed JC: Bax directly induces release of cytochrome $c$ from isolated mitochondria. Proc Natl Acad Sci USA 95: 4997-5002, 1998.

8. Kluck RM, Bossy-Wetzel E, Green DR and Newmeyer DD: The release of Cytochrome $c$ from mitochondria: a primary site for Bcl-2 regulation of apoptosis. Science 275: 1132-1136, 1997.

9. Yang J, Liu X, Bhalla K, et al: Prevention of apoptosis by Bcl-2: release of Cytochrome $c$ from mitochondria blocked. Science 275: 1129-1132, 1997.

10. Millenbaugh NJ, Roth C, Sypniewska R, et al: Gene expression changes in the skin of rats induced by prolonged $35 \mathrm{GHz}$ millimeter-wave exposure. Radiat Res 169: 288-300, 2008.

11. Zhadobov M, Sauleau R, Le Coq L, et al: Low-power millimeter wave radiations do not alter stress-sensitive gene expression of chaperone proteins. Bioelectromagnetics 28: 188-196, 2007.

12. Szabo I, Kappelmayer J, Alekseev SI and Ziskin MC: Millimeter wave induced reversible externalization of phosphatidylserine molecules in cells exposed in vitro. Bioelectromagnetics 27 : 233-244, 2006.

13. Wu GW, Liu XX, Wu MX, et al: Experimental study of millimeter wave-induced differentiation of bone marrow mesenchymal stem cells into chondrocytes. Int J Mol Med 23: 461-467, 2009.

14. Li XH, Du M, Liu XX, et al: Millimeter wave treatment inhibits NO-induced apoptosis of chondrocytes through the p38MAPK pathway. Int J Mol Med 25: 393-399, 2010.

15. The Ministry of Science and Technology of the People's Republic of China: Guidance Suggestions for the Care and Use of Laboratory Animals, 2006.

16. Lin JM, Chen YQ, Wei LH, et al: Hedyotis Diffusa Willd extract induces apoptosis via activation of the mitochondrion-dependent pathway in human colon carcinoma cells. Int $\mathrm{J}$ Oncol 37: 1331-1338, 2010.

17. Tonomura H, Takahashi KA, Mazda O, et al: Glutamine protects articular chondrocytes from heat stress and NO-induced apoptosis with HSP70 expression. Osteoarthritis Cartilage 14: 545-553, 2006.

18. Heinegard D, Bayliss M, and Lorenzo P: Biochemistry and metabolism of normal and osteoarthritic cartilage. In: Osteoarthritis. Brandt KD, Doherty M, and Lohmander LS (eds). Oxford University Press, New York, NY, pp74-84, 1998.

19. Pritzker K: Pathology of osteoarthritis. In: Osteoarthritis. Brandt KD, Doherty $M$ and Lohmander LS (eds). Oxford University Press, New York, NY, pp50-61, 1998.

20. Kim HA and Blanco FJ: Cell death and apoptosis in osteoarthritic cartilage. Curr Drug Targets 8: 333-345, 2007.

21. Li XH, Du M, Liu XX, et al: Millimeter wave treatment promotes chondrocyte proliferation by up-regulating the expression of cyclindependent kinase 2 and cyclin A. Int J Mol Med 25: 393-399, 2010.

22. Mantymaa P, Siitonen T, Guttorm T, et al: Induction of mitochondrial manganese superoxide dismutase confers resistance to apoptosis in acute myeloblastic leukaemia cells exposed to etoposide. Br J Haematol 108: 574-581, 2000.

23. Korper S, Nolte F, Rojewski MT, Thiel E and Schrezenmeier H: The $\mathrm{K}^{+}$channel openers diazoxide and NS1619 induce depolarization of mitochondria and have differential effects on cell $\mathrm{Ca}^{2+}$ in CD34 ${ }^{+}$cell line KG-1a. Exp Hematol 31: 815-823, 2003.

24. Cossarizza A, Baccarani-Contri M, Kalashnikova G and Franceschi C: A new method for the cytofluorimetric analysis of mitochondrial membrane potential using the J-aggregate forming lipophilic cation 5,5',6,6'-tetrachloro-1,1',3,3' tetraethylbenzimidazolylcarbocyanine iodide (JC-1). Biochem Biophys Res Commun 197: 40-45, 1993.

25. Smiley ST, Reers M, Mottola-Hartshorn C, et al: Intracellular heterogeneity in mitochondrial membrane potentials revealed by a J-aggregate forming lipophilic cation JC-1. Proc Natl Acad Sci USA 88: 3671-3675, 1991.

26. Eskes R, Desagher S, Antonsson B and Martinou JC: Bid induces the oligomerization and insertion of bax into the outer mitochondrial membrane. Mol Cell Biol 20: 929-935, 2000. 